\title{
Vibration, synchronization and localization of three-bladed rotor: theoretical and experimental studies
}

\author{
Z. Szmit ${ }^{1, a}(\mathbb{D}$ \\ ${ }^{1}$ Department of Applied Mechanics, Faculty of Mechanical Engineering, Lublin University of Technology, Lublin, Poland
}

Received 10 April 2021 / Accepted 7 July 2021 / Published online 2 August 2021

(C) The Author(s) 2021

\begin{abstract}
Numerical and experimental methods in free and forced vibrations of the rotating structure consisting of the rigid hub and three flexible beams are considered. Firstly, the system of four mutually coupled dimensionless differential governing equations is presented and then forced response of the system as well as synchronization phenomenon are investigated. Next, the finite elements method is used to design the rotating structure and analyse complex dynamic response. During the numerical calculations symmetric, as well as de-tuned rotor are analyzed. Finally, results obtained from ordinary differential equations and numerical simulations are compared with experimental tests.
\end{abstract}

\section{Introduction}

Rotating structures are important machine components, which are widely used in several industrial applications. The most common ones are helicopter rotors, wind turbines, fans, airplane propellers etc. Second well-known group of rotating structures are rigid disks combined with a series of beam elements. As the examples one can mention, turbojet aircraft engines, gas and steam turbines and axial compressors.

The Hamilton's principle is usually used to derive governing equations of motion for rotating structures. Based on equations the test of system stability or individual blades motion synchronization can be solved in analytical and numerical way $[3,4,17]$. The dynamics of a hub-beam system is analyzed in [12]. Authors performed parametric studies with respect to the laminate orientation angle as well as different regular driving torque. The parametric studies for a rotating hub with Timoshenko beam element as well as saturation control is performed in $[23,24]$. Different approach for the rotating system is presented in [22]. Authors focused on slender beam model with tip mass and based the calculations on extended Bernoulli-Euler theory. The multiple time scale method is used for analytical calculation as well as forced vibration and influence of parameters as tip mass are studied. Extension of the work on a rotating hub-beam model is shown in [21], where authors present different types of control and reduce beam oscillations by applying active elements on beam. The free and forced nonlinear vibrations are studied and have shown hardening and softening phenomena.

In the 17 th century a Dutch researcher Christiaan Huygens discovered the synchronization phenomenon

\footnotetext{
a e-mail: z.szmit@pollub.pl (corresponding author)
}

in case of a pendulum clock hanging on the same beam [8]. Next in the $21^{\text {st }}$ century similar experiment has been repeated and extensively described in [9]. Since the discovery of the synchronization phenomenon, many examples have been given in the mechanical, electrical and biological systems [2]. Because of the way how phenomenon was discovered, synchronization is closely connected with pendulums. In the papers $[1,10,16]$ results for synchronization phenomenon in case of rotating parametric pendulums are presented, whereas, the problem of rotational motion in different and the same direction of the pendulums is described in [5]. Furthermore, co- and counter-rotating coupled spherical pendulums is studied in [28]. The coupled in-phase synchronization in case of mechanical oscillators array is performed in [27]. Moreover, the chimera state of coupled pendulums, which is strictly connected with synchronization phenomenon is presented in [6,7]. Most of the scientific papers and all mentioned above, which give a big contribution to the knowledge of synchronization, focus on the pendulum dynamics in a vertical plane. The nonlinear system composed of two pendulums fixed to rotating hub in a horizontal plane are investigated in [26]. Authors analysed the synchronization phenomenon, as well as transitions through resonances considering the influence of the hub inertia. They found chaotic oscillations in the system and demonstrated the path, which leads to chaos.

In the present contribution, the numerical and experimental studies are introduced. The numerical studies are divided into two parts, firstly resonance curves for a rotating system composed of a rigid hub and three slander beams are drawn based on the ordinary differential equations of motion. Secondly, the Finite Elements Method is used to analyse dynamics of the rotor. Then, the results from numerical calculations are compared 
with the experimental tests. During the experimental studies, the dynamics of the system and synchronization phenomenon are analyzed.

\section{Model and equations of motion}

Rotor consisting of three slender and elastic composite blades attached to the rigid hub is investigated. The rotor plane $\left(X_{0}, Y_{0}\right)$ coincides with flapwise bending plane of blades $(x, z)$. The system is excited by external torque $T$, which induce rotation around a fixed frame vertical axis $C Z_{0}$. The hub may oscillate in the vicinity of one position or rotate, and current angular position of the hub is denoted by an angle $\psi(t)$. Each beam is made of an eighteenlayered laminate of unidirectional graphite-epoxy prepreg material. The applied specific stacking sequence $\left(0 /-60 / 60 / 0 /-60 / 60_{3} /-60_{2} / 0_{2} /-60 / 0_{2} / 60_{2} /-60\right)$ results in full isotropic composite material behaviour, according to [20] (Fig. 1).

The partial differential equations of motion of the rotating structure have been derived according to the expended Hamilton's principle. In the formulation the lead-lag displacement, transverse shear and profile twist of the beams are taken into account. The detailed description of the derivation procedure is given in [25]. Next, partial differential equations have been transformed to a dimensionless ordinary differential form by adopting the Galerkin method. In this case, the Galerkin procedure is applied for the first natural mode. The set of coupled nonlinear ordinary differential equations of motion presents as:

$$
\begin{aligned}
& \left(J_{\mathrm{h}}+\sum_{i=1}^{3} J_{\mathrm{b} i}+\sum_{i=1}^{3} \alpha_{\mathrm{h} i 2} q_{i}^{2}\right) \ddot{\psi}+\zeta_{\mathrm{h}} \dot{\psi} \\
& +\sum_{i=1}^{3}\left(\alpha_{\mathrm{h} i 1} \ddot{q}_{i}+\alpha_{\mathrm{h} i 3} q_{i} \dot{q}_{i} \dot{\psi}\right)=\mu(\tau) \\
& \ddot{q}_{1}+\zeta_{1} \dot{q}_{1}+\alpha_{12} \ddot{\psi}+\left(\alpha_{11}+\alpha_{13} \dot{\psi}^{2}\right) q_{1}+\alpha_{14} q_{1} \dot{q}_{1} \dot{\psi}=0 \\
& \ddot{q}_{2}+\zeta_{2} \dot{q}_{2}+\alpha_{22} \ddot{\psi}+\left(\alpha_{21}+\alpha_{23} \dot{\psi}^{2}\right) q_{2}+\alpha_{24} q_{2} \dot{q}_{2} \dot{\psi}=0 \\
& \ddot{q}_{3}+\zeta_{3} \dot{q}_{3}+\alpha_{32} \ddot{\psi}+\left(\alpha_{31}+\alpha_{33} \dot{\psi}^{2}\right) q_{3}+\alpha_{34} q_{3} \dot{q}_{3} \dot{\psi}=0,
\end{aligned}
$$

where $J_{\mathrm{h}}$ and $J_{\mathrm{b} i}$ describe the mass moment of inertia of the hub and each beam, respectively. These values are relative to beam 1 . The parameters $\zeta_{\mathrm{h}}$ and $\zeta_{i}$ $(i=1,2,3)$ take a role of viscous damping coefficients for the hub and each beam. The hub damping coefficient has been set at 0.1 and the approximate values of $\zeta_{i}$ equals 0.04 ratio of the beam natural frequency $\omega_{0 i}$. The external driven torque is applied to the hub and is denoted by $\mu(\tau)$, where $\tau$ is a dimensionless time. The external excitation is expressed as analytical function $\mu=\mu_{0}+\rho \sin \omega t$. Two types of rotor have been analyzed-fully symmetric and slightly de-tuned. First, fully symmetric model with all the same beams has been investigated. All the parameters $\alpha_{\mathrm{h} i j}(j=1,2,3)$ and $\alpha_{i k} k=1, \ldots, 4$ are obtained during the Galerkin procedure of reduction and are presented in Table 1.

Studying the set of Eq. (1) it is easy to observe that each beam equation (last three equations in the set (1)) is coupled by mass moment of inertia with the hub equation. In case when angular velocity is constant, all equations are uncoupled. On the other hand, when the angular velocity is not constant, all equations are coupled and the nonlinear terms as well as Coriolis forces are taken into account in the dynamics of the structure. To investigate the dynamics of the fully symmetric rotor, the numerical calculations have been performed based on the Eq. (1) and coefficients from the Table 1. The resonance curves have been drawn for the angular velocity of the hub $\dot{\psi}$ and displacement of beams $q_{1}, q_{2}, q_{3}$ - see Fig. 2. In both cases the amplitude of excitation is equal $\rho=0.03$ and the constant component of the torque $\mu_{0}=0$. One can observe, that the rotor is fully symmetric, just a single resonance zone is presented with the peak resonance around $\omega \approx 3.6$. Moreover, at the resonance frequency, all beams are fully synchronized in-phase, while the angle of rotation of the hub is synchronized with small locked phase, which is a result of damping in the system - see Fig. 3.

Second, slightly de-tuned model has been analyzed, where only one beam (beam No. 2) is $10 \%$ thicker. For this purpose the Galerkin procedure of reduction is performed and new parameters for equations of motion (1) are obtained and presented in Table 2. To understand better and find differences betweens two analyzed models, the dynamics of the de-tuned rotating model the numerical calculations have been performed based on the Eq. (1) and coefficients from the Table 2. Similar like in the first case, the resonance curves have been plotted for the angular velocity of the hub $\dot{\psi}$ and displacement of beams $q_{1}, q_{2}, q_{3}$-see Fig. 4. Graphs are performed for the same parameters of excitation. On the other hand,in the case of de-tuned rotor two resonance zones are presented with the peaks resonance around $\omega \approx 3.6$ and $\omega \approx 3.9$. At the first resonance frequency vibrations are localized in the two the same beams, while in the second resonance peak vibrations are localized in the thicker beam (beam No. $2-q_{2}$ ).

Moreover, in both resonance peaks all beams Nos. 1 and 3 are fully synchronized, while for the thicker beam synchronization with locked phase take place (Fig. 5). The angle of rotation of the hub in the resonance zones are synchronized in anti-phase with the beam in which vibrations are localized. For the frequency $\omega=3.6$ motion of the hub is synchronized in anti-phase with beams Nos. 1 and 3, whereas, at the frequency $\omega=3.9$ with de-tuned beam.

\section{Finite element simulations}

The finite element model of the of three blades-hub structure is built on the $X-Y$ plane. A reference point 
Fig. 1 Model of the rotating hub with three slender blades

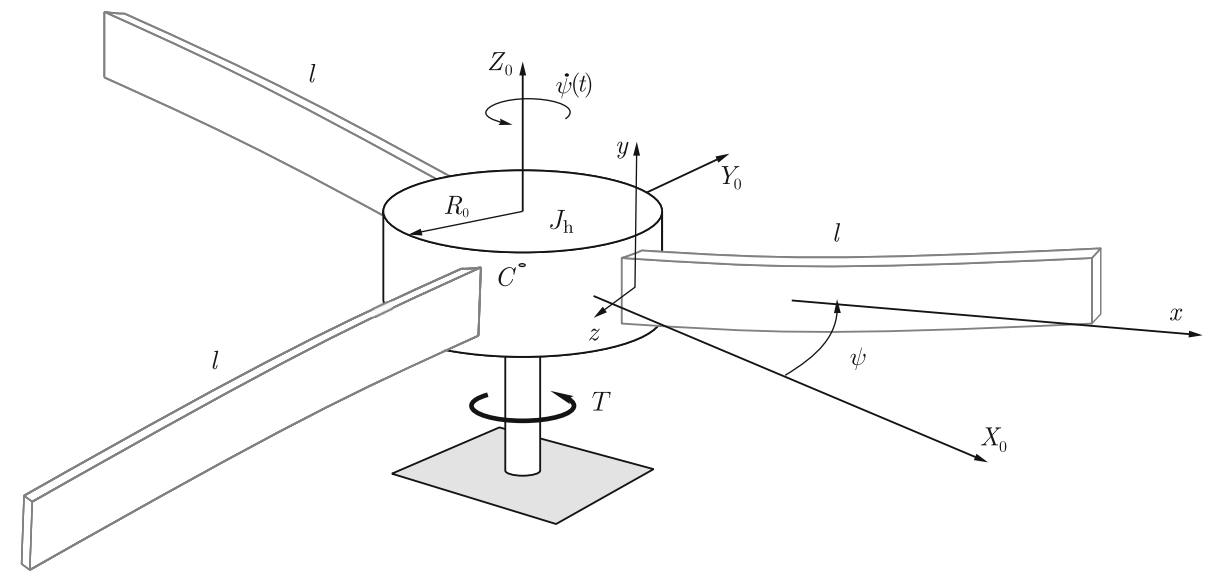

Table 1 The values of dimensionless coefficient present in the ordinary differential equations of motion-symmetric rotor

\begin{tabular}{lllll}
\hline Beams & $\alpha_{i 1}=12.36502$ & $\alpha_{i 2}=1.80558$ & $\alpha_{i 3}=0.37094$ & $\alpha_{i 4}=-1.55324$ \\
Hub & $\alpha_{\mathrm{h} i 1}=0.05035$ & $\alpha_{\mathrm{h} i 2}=-0.03823$ & $\alpha_{\mathrm{h} i 3}=-0.07646$ & \\
\hline
\end{tabular}

Fig. 2 Resonance curves of angular velocity of the hub $\dot{\psi}$ (left) and displacement of beams $q_{1}, q_{2}, q_{3}$ (right); the amplitude of excitation $\rho=0.03$ - symmetric model

Fig. 3 Time histories of individual beams ends and angle of hub rotation at the resonance zone-symmetric model
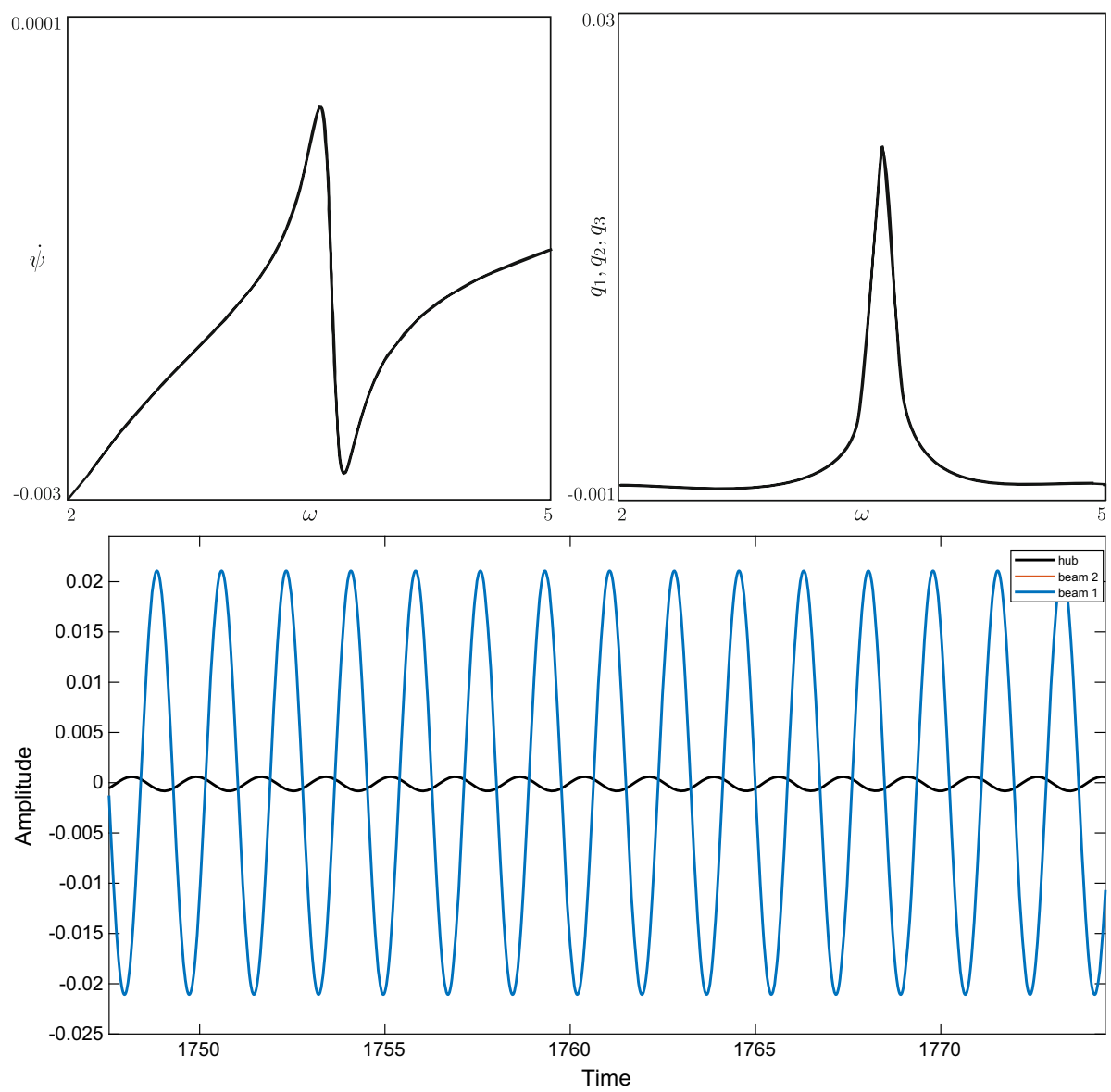

Table 2 The values of dimensionless coefficient present in the ordinary differential equations of motion- de-tuned model

\begin{tabular}{lllll}
\hline Beams 1 & $\alpha_{11}=12.36502$ & $\alpha_{12}=1.80558$ & $\alpha_{13}=0.37094$ & $\alpha_{14}=-1.55324$ \\
Beams 2 & $\alpha_{21}=14.96158$ & $\alpha_{22}=1.80505$ & $\alpha_{23}=0.37089$ & $\alpha_{24}=-1.55296$ \\
Hub & $\alpha_{\mathrm{h} i 1}=0.05035$ & $\alpha_{\mathrm{h} i 2}=-0.03823$ & $\alpha_{\mathrm{h} i 3}=-0.07646$ & \\
\hline
\end{tabular}


Fig. 4 Resonance curves of angular velocity of the hub $\dot{\psi}$ (left) and displacement of beams $q_{1}$-black, $q_{2}$-red, $q_{3}$-black (right); the amplitude of excitation $\rho=0.03$ - de-tuned model
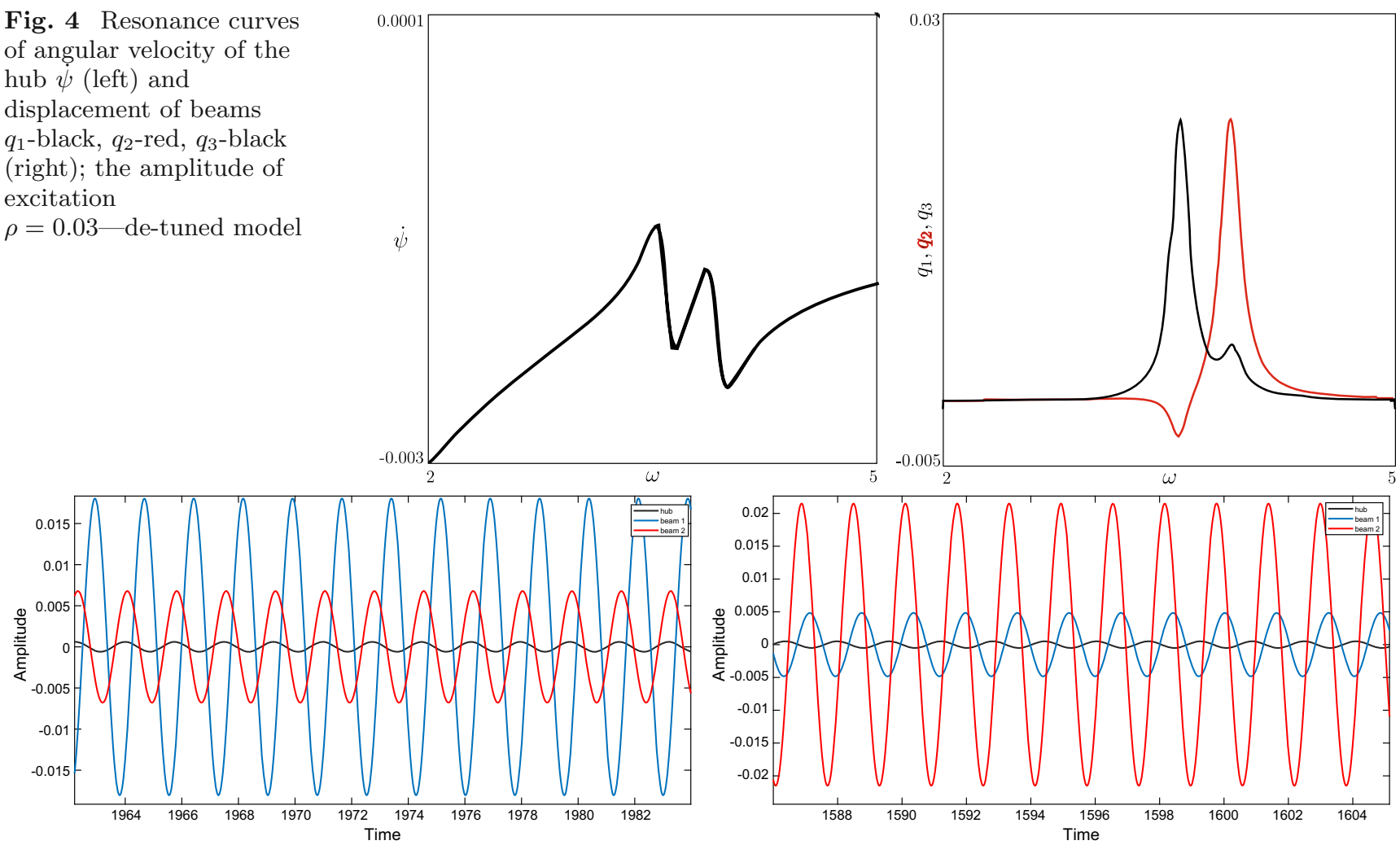

Fig. 5 Time histories of individual beams and angle of hub rotation at the resonance zones for the frequency $\omega=3.6$ (left) and $\omega=3.9$ (right) - de-tuned model

$R P$ lies at the origin of coordinate system $X_{0}=0$, $Y_{0}=0, Z_{0}=0$ and determines the axis of rotation, which is perpendicular to the plane of rotating disk. The mass moment of inertia of the hub is reduced to the single point (RP) and involves the drive shaft, blade grips, bearings and movable electric brushes of the forthcoming experimental setup, see Sect. 4. Having regard to the radius of the hub $R_{h}=120 \mathrm{~mm}$, three blades of the same length $L=595 \mathrm{~mm}$, distributed by the same angle $2 / 3 \pi$ are fixed via kinematic coupling to the single point hub. Using link definition, motion of the hub can act on the beams as well as dynamics of beams influence on the hub response, and note that the preset angle can be arbitrary modified. Mechanical properties of the rectangular cross-section composite beams $(b \times h=34 \times 0.9$ $\mathrm{mm}$ ) are simplified by homogeneous Young's modulus $E=55722.51 \mathrm{MPa}$, Poisson's ratio 0.36 and uniform density $\rho=1.35 * 10^{-9}$ tone $/ \mathrm{mm}^{3}$. The beam, as a substructure of the rotor, consists of 100 equal-length, B32-type finite elements. In the boundary conditions, the freedom of the hub movement in $X_{0}, Y_{0}, Z_{0}$ directions as well as rotations about $X_{0^{-}}$and $Y_{0^{-}}$-axes are restrained, moreover, external excitation is not considered here.

To impose linear modal solutions of the complex structure the Lanczos Eigensolver is used in the first 2 modes determination. In the numerical outcomes we get a set of natural frequencies and associated mode shapes wherein two different dynamical responses can be found: (a) dynamics of the rotor as the hub with beams and (b) frozen hub with modal coupling of beam shapes that balance with respect to the center of rotation. In this paper, only dynamics of the rotor is analyzed because only this part can be validated during the experimental tests. Four types of the rotor are investigated by the finite elements method: (a) fully symmetric model, (b) rotor with one beam 10\% thicker, (c) rotating model with one beam $10 \%$ shorter and finally (d) structure with one beam 10\% longer.

Let us start with the fully symmetric model, in Fig. 6 behaviour of the whole rotor in the first resonance frequency equal $5.9537 \mathrm{~Hz}(37.4086 \mathrm{rad} / \mathrm{s})$ is presented. One can observe, that all three beams are moving in the same direction. The time histories show that the rotor in the first resonance zone is fully synchronized, while beams are synchronized in magnitude and phase with each other and stay in anti-phase with respect to motion of the hub.

In the second resonance zone, at frequency equal $19.408 \mathrm{~Hz}(121.944 \mathrm{rad} / \mathrm{s})$, similar dynamics of the beams can be observed-see Fig. 7. The difference between the first and second natural frequencies is easier to find at the time series. It is clearly seen that motion of whole rotor, three beams and the hub is fully synchronized. Beams are synchronous in magnitude and phase, while the motion of the hub is synchronized in phase with beams.

The second investigated model has two the same beams and one $10 \%$ thicker. The frequency of the first resonance equal $6.2564 \mathrm{~Hz}(39.3101 \mathrm{rad} / \mathrm{s})$ and motion 

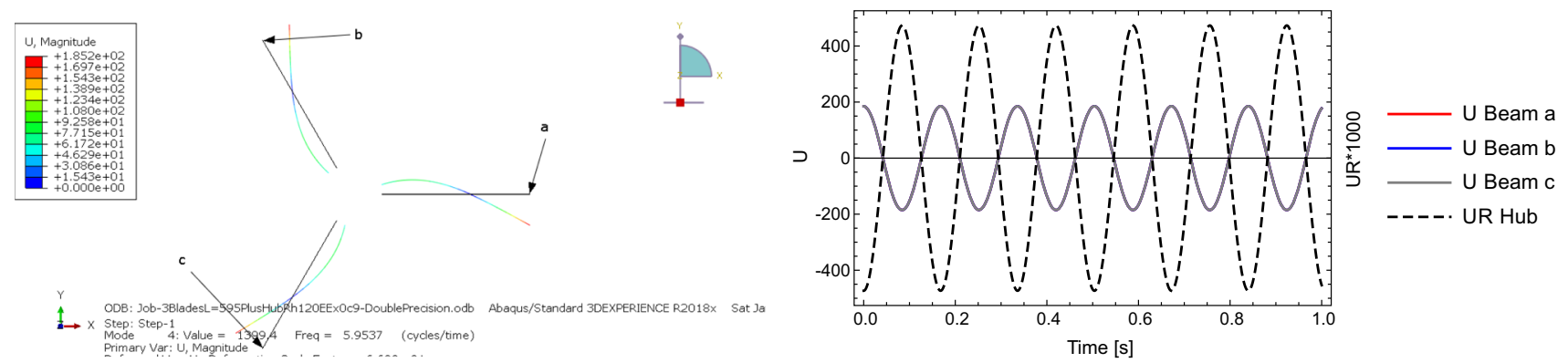

Fig. 6 Beams displacement in the first resonance zone with frequency equal $5.9537 \mathrm{~Hz}$ - print screen from Abaqus software and time histories of individual beams ends and angle of hub rotation-symmetric model
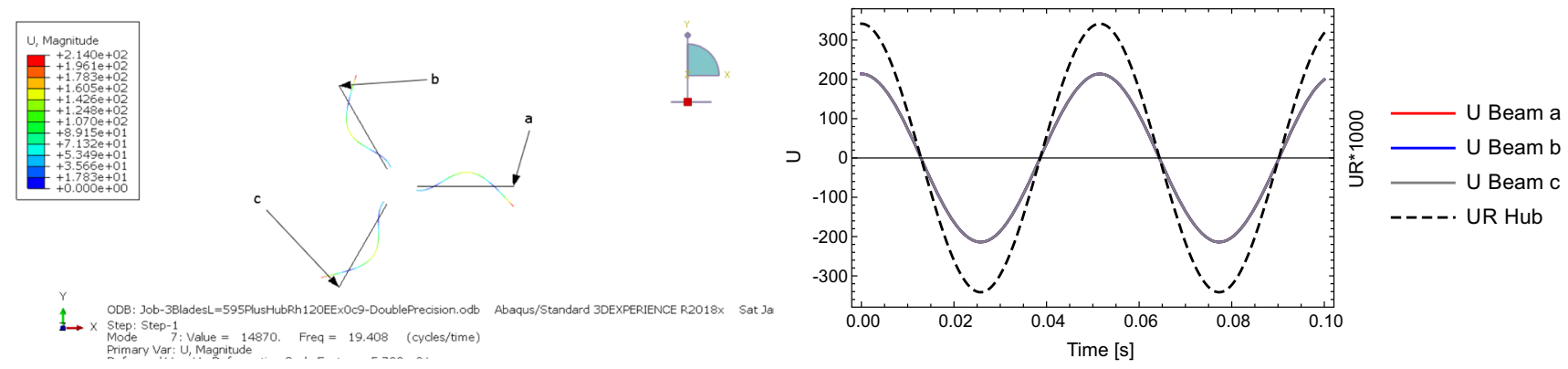

Fig. 7 Beams displacement in the second resonance zone with frequency equal $19.408 \mathrm{~Hz}$ - print screen from Abaqus software and Time histories of individual beams and angle of hub rotation -symmetric model
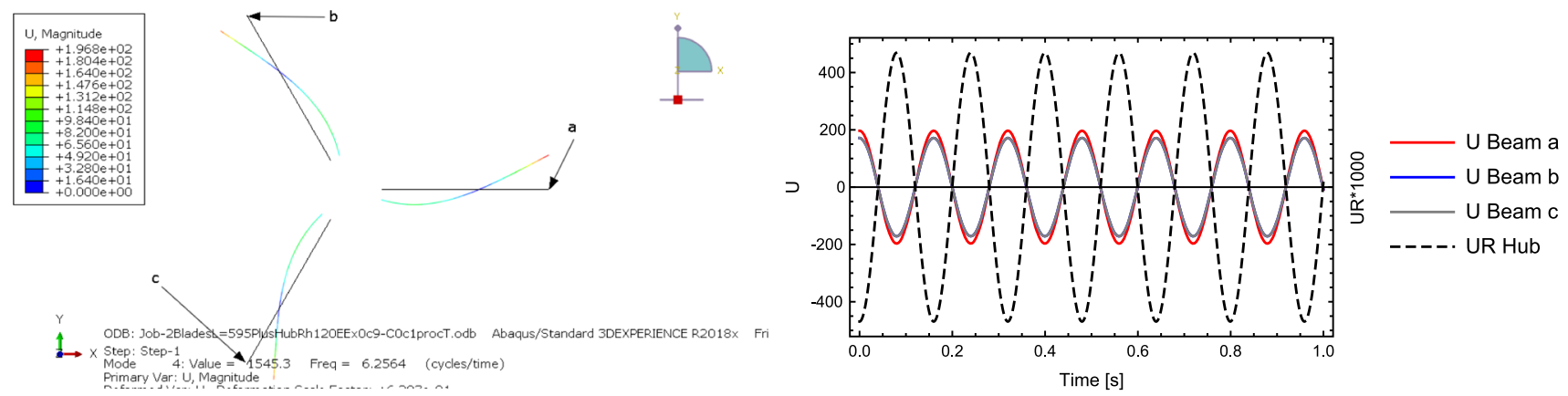

Fig. 8 Beams displacement in the first resonance zone with frequency equal $6.2564 \mathrm{~Hz}$ - print screen from Abaqus software and time histories of individual beams and angle of hub rotation-model with one thicker beam

of the beams is synchronous in phase with slight difference of the magnitude - see Fig. 8. Moreover, the synchronization in the anti-phase of the hub with respect to the beams is observed.

At the second resonance zone $(20.5750 \mathrm{~Hz})$, the vibrations are localized in the one thicker beam (beam a). Furthermore, in Fig. 9 the synchronization scenario is similar to the motion of the rotor in the first resonance frequency.

Next analyzed model has one beam $10 \%$ shorter, while the cross-section of all beams is the same. The first resonance appeared at frequency equal $6.2613 \mathrm{~Hz}$ (39.3409 rad/s) and show synchronous motion of all the beams with small difference of the magnitude between the same beams and de-tune one. Motion of the hub with respect to the beams is synchronized in antiphase-see Fig. 10.
At the frequency $21.891 \mathrm{~Hz}(137.545 \mathrm{rad} / \mathrm{s})$ second resonance is observed. In this case, the fully synchronization in-phase can be observed between all beams and the motion of the hub. In the time series (Fig. 11) the difference of the magnitude between de-tune beam and nominal beams is noticeable.

The last investigated rotor performed in the finite element method has $10 \%$ longer beam then nominal ones in the symmetrical model (Fig. 12).

At first as well at second resonance zone beams between each other are synchronized in-phase with the difference of the magnitude between nominal blades and de-tune beam. Moreover, in both zones, motion of the hub with respect to the beams is synchronized in antiphase. One can observe, that only in this model the vibrations are localized in the two same beams not in the de-tune one (Fig. 13). 

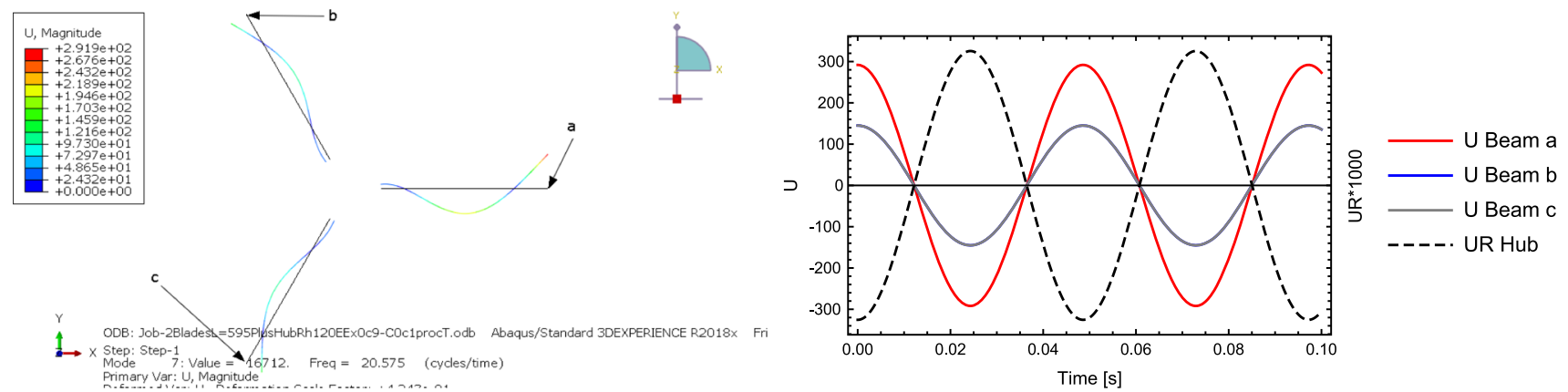

Fig. 9 Beams displacement in the second resonance zone with frequency equal $20.5750 \mathrm{~Hz}$ - print screen from Abaqus software and time histories of individual beams and angle of hub rotation - model with thicker beam
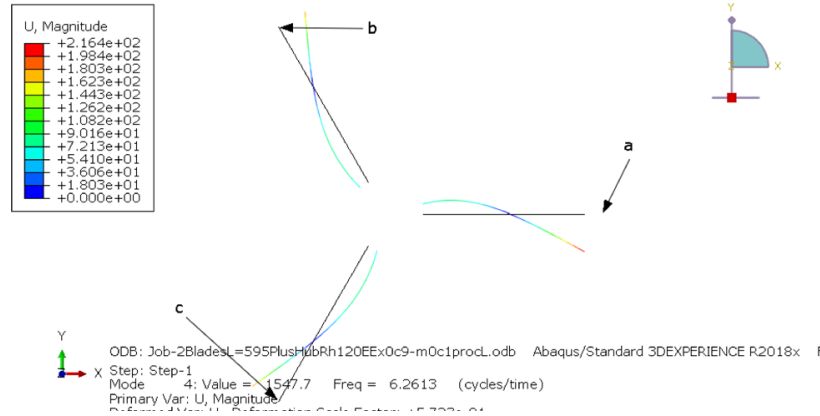

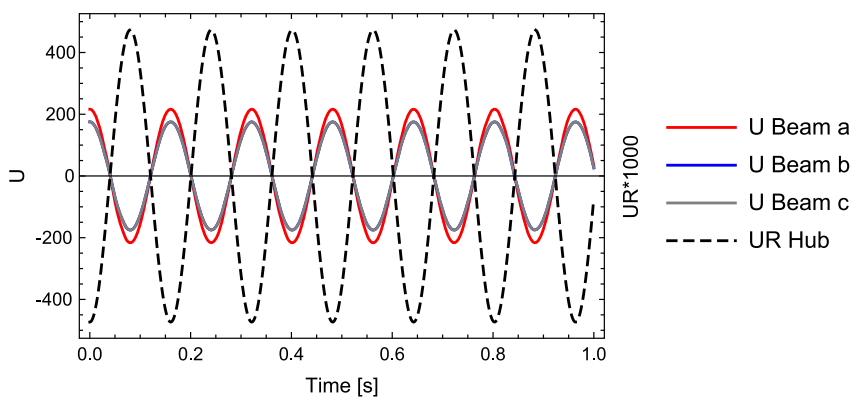

Fig. 10 Beams displacement in the first resonance zone with frequency equal $6.2613 \mathrm{~Hz}$ - print screen from Abaqus software and time histories of individual beams and angle of hub rotation-model with shorter beam
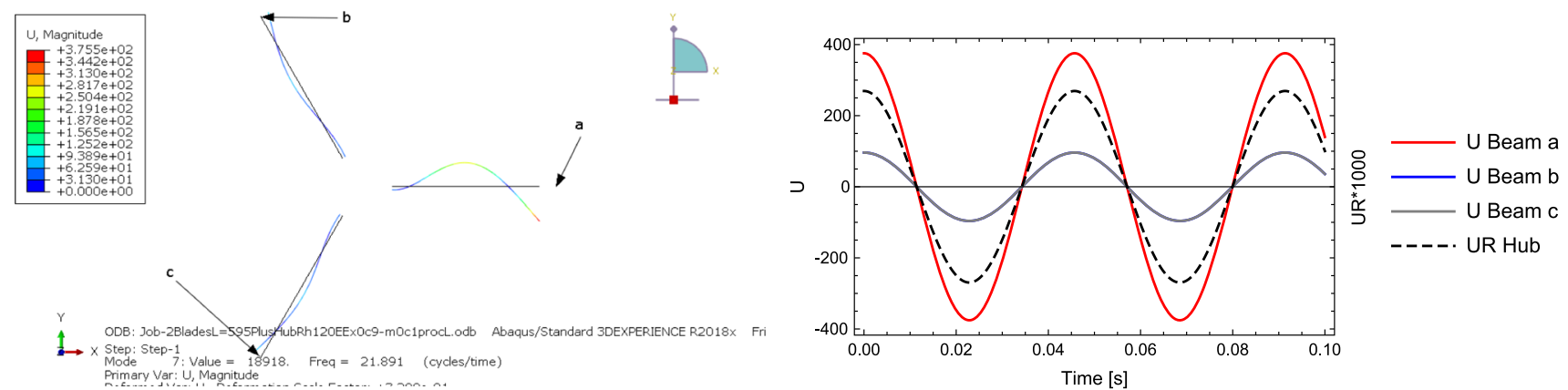

Fig. 11 Beams displacement in the second resonance zone with a frequency equal $21.891 \mathrm{~Hz}$ - print screen from Abaqus software and time histories of individual beams and angle of hub rotation-model with shorter beam
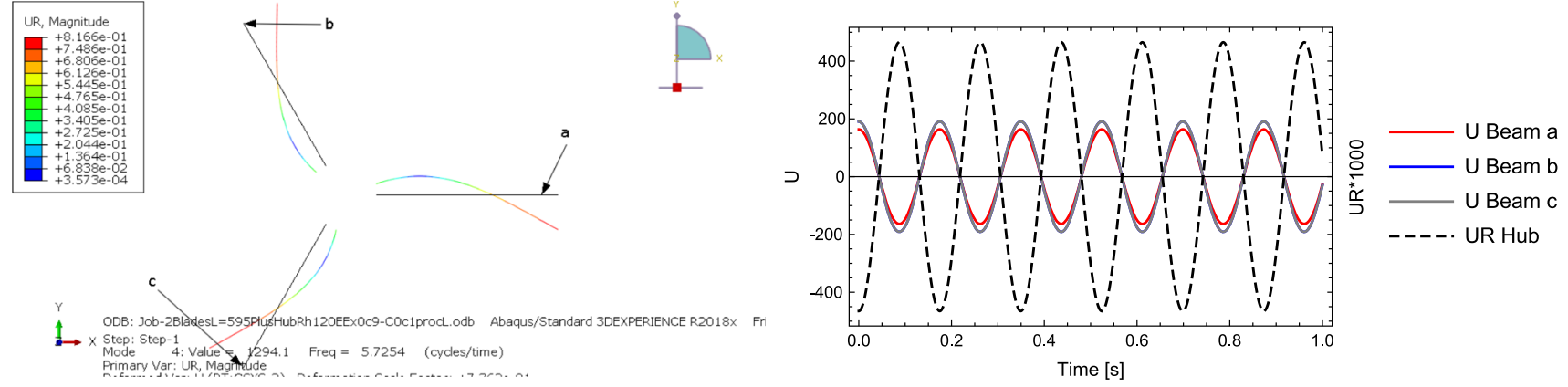

Fig. 12 Beams displacement in the first resonance zone with frequency equal $5.7254 \mathrm{~Hz}$ - print screen from Abaqus software and time histories of individual beams and angle of hub rotation - model with longer beam 

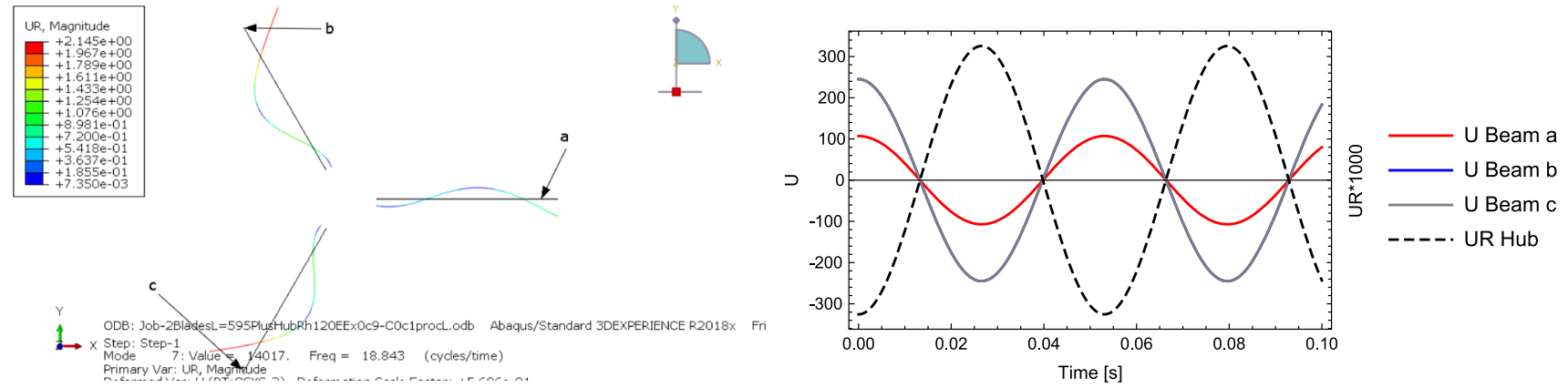

Fig. 13 Beams displacement in the second resonance zone with frequency equal $18.843 \mathrm{~Hz}$ - print screen from Abaqus software and time histories of individual beams and angle of hub rotation - model with longer beam

\section{Experimental tests}

Experimental studies have been conducted on the laboratory stand, which was designed for scientific research in the Department of Applied Mechanics at the Lublin University of Technology. The photo of the laboratory stand is shown in Fig. 14. The rotor stand consist of a rotating rigid hub and three composite beams. Each beam has a length of $595 \mathrm{~mm}$ and has the rectangular cross section of $34 \times 0.9 \mathrm{~mm}$ and is made of an eighteen-layered laminate of graphite-epoxy prepreg material, which is applied in stacking sequence $\left(0 /-60 / 60 / 0 /-60 / 60_{3} /-60_{2} / 0_{2} /-60 / 0_{2} / 60_{2} /-60\right)$. The strain gauges are applied to beams and the signal from them is sent to computer software by wireless module. The radius of the hub is $120 \mathrm{~mm}$. There are boards with electronic circuits that allow to transmit signals from strain gauges. The rotating hub is exited by DC motor with a power of $0.96 \mathrm{~kW}$. The helical clutch is used to combine the DC motor with the hub, also the set of nine slip rings is applied for the the transmission of measuring and control signals.

On the described test stand, the experiments with constant angular velocity or excitation of unlimited signal, as well as excitation with different frequencies are possible. This contribution is focused on the experiments, where the frequency of excitation is changed. For a closer examination of the synchronization phenomenon between beams and the hub, during the experimental studies high-speed camera is used.

\subsection{Natural frequency}

First, the linear dynamic response of the cantilever beam has been tested. The experiment has been performed on the laboratory stand and the non-contact scanning laser vibrometer Polytec PSV-500 has been used to carry out the values of natural frequencies $[11,14,15]$. Two types of exam have been executed, by force impulse and by the initial deformation about the first mode of the beam.

The first natural frequency of the beam is shown in Fig. 15 and is equal $2.625 \mathrm{~Hz}$ (16.4934 rad/s), whereas, in Fig. 16 is presented second natural frequency and is equal $16.55 \mathrm{~Hz}(103.987 \mathrm{rad} / \mathrm{s})$. Both types of exam

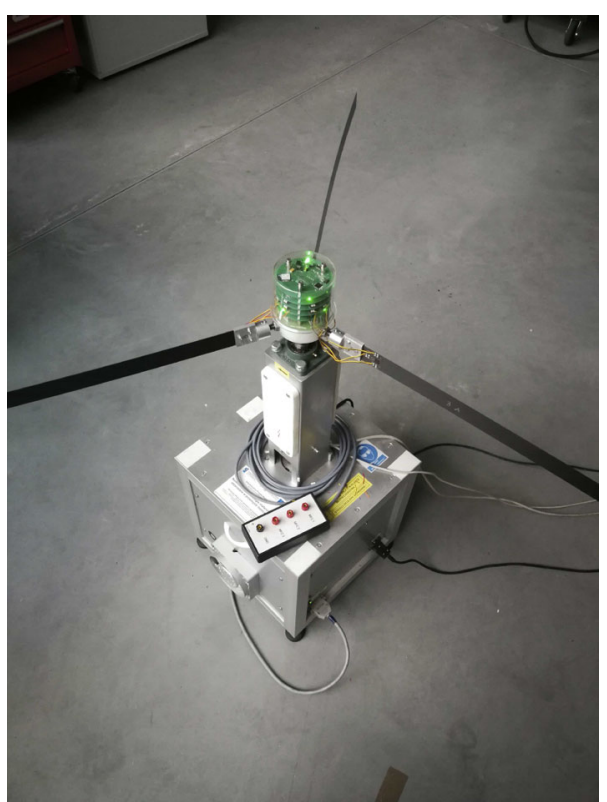

Fig. 14 Rotor laboratory stand

during the modal analysis have shown similar results, which proves that tests are performed correctly.

\subsection{Forced vibrations}

The second part of the experimental studies has been labor-intensive and time-consuming. In this case, the forced vibrations have been analysed at hub-beams laboratory stand, the frequency of excitation has been changed from $1.0 \mathrm{~Hz}(6.283185307 \mathrm{rad} / \mathrm{s})$ with step 0.1 to $22 \mathrm{~Hz}(138.230076758 \mathrm{rad} / \mathrm{s})$. The experimental stand has been driven for each value of the excitation frequency at least one minute. Then, based on the signals from the strain gauges time histories have been prepared. The amplitudes have been interpreted from the time series and the amplitude-frequency graph has been drown - see Fig. 17.

For the moderately large excitation applied to the hub, at the resonance curve from the experimental studies two main resonance zones appeared, with resonance peaks at frequency $5.9 \mathrm{~Hz}$ and $19.7 \mathrm{~Hz}$. Moreover, tests performed at laboratory stand show two smaller peaks 
Fig. 15 First natural frequency of beam-modal analysis

Fig. 16 Second natural frequency of beam-modal analysis

Fig. 17

Amplitude-frequency response-experimental studies
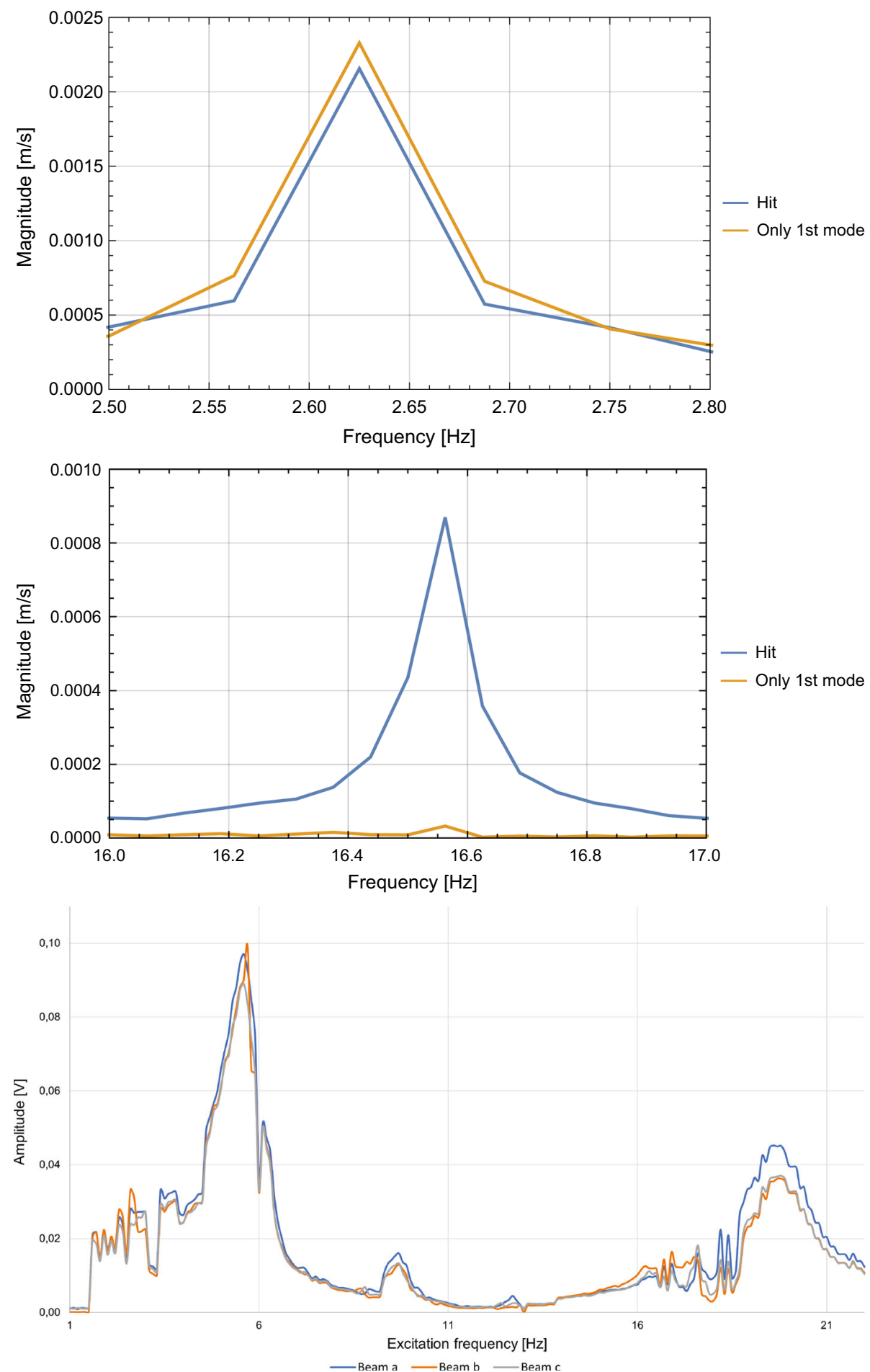

at frequencies $9.7 \mathrm{~Hz}$ and $12.7 \mathrm{~Hz}$, which will be analyzed in the future work. For a better understanding of the synchronization phenomenon in the resonance zones during the experimental studies time histories and movies from high-speed camera have been performed. From the time series for the first and second resonance peaks (see Figs. 18, 19) one can see that, all beams are synchronized in phase with each other. However all beams have the same natural frequency and geometry, small difference between them appeared, in a result from the composite beam production process. For that reason small differences in the magnitude at the time histories can be observed. Considering only time histories it is not possible define relations between motion of the beams and the hub, thus the high-speed camera has been used. The movies of the rotor at first resonance zone show that all the beams are moving in the same direction, but the hub moves in the opposite direction-Fig. 20. Based on the films, it can be observed that beams and the hub are synchronized in 
Fig. 18 Time histories of individual beams for frequency 5.9

$\mathrm{Hz}$ - experimental studies

Fig. 19 Time histories of individual beams for frequency 19.7 $\mathrm{Hz}$ - experimental studies
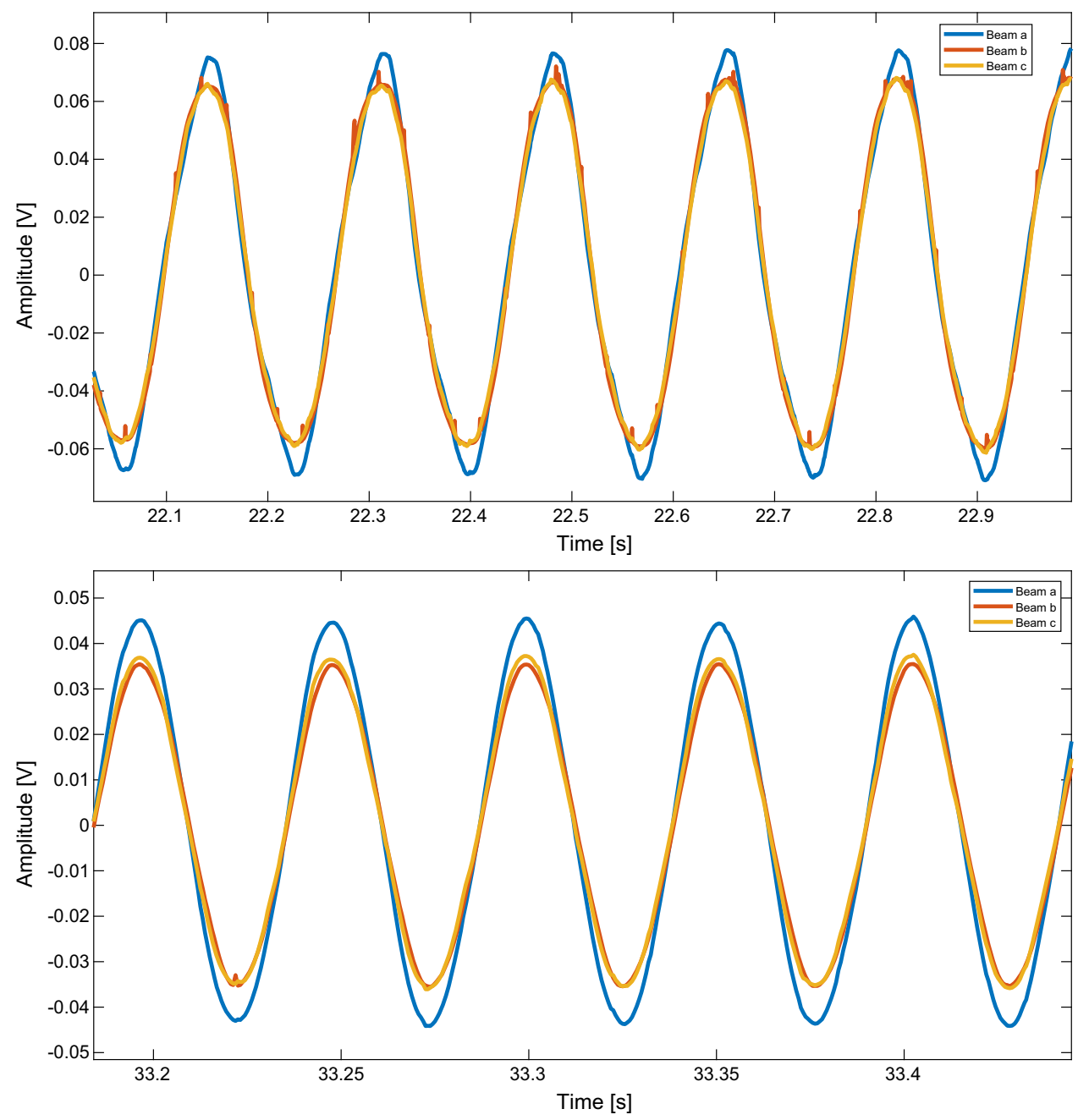

anti-phase. When the rotor is excited by the second resonance frequency beams and the hub rotated in the same direction, it means that the beams and the hub are fully synchronized in phase (Fig. 21).

\section{Final remarks}

The presented paper considers numerical and experimental studies on a rotating structure consisting of the rigid hub and three composite beams. The numerical studies based on the ordinary differential equations of motion as well as on the finite elements model analyzed in Abaqus software. Additionally, in the numerical calculation the symmetric as well as de-tuned models are investigated. The results from numerical calculations and simulations of the symmetric rotor are compared with the experimental test. During the experimental studies the natural vibration and forced vibration are performed. The synchronization phenomenon on every step of calculation is considered. The results from simulations of the symmetric model in Abaqus software and from experimental tests are showing very good agreement, qualitatively and quantitatively. First resonance frequency is $5.9 \mathrm{~Hz}$ in both studies, while the second resonance frequency from finite elements method is 19.4 $\mathrm{Hz}$ and for experiment is $19.7 \mathrm{~Hz}$. These very close to each other numbers prove that the FE model is a faithful reflection of the actual laboratory stand. All results from calculations of symmetrical rotor showed the same scenario for synchronization phenomenon. At the first resonance zone, full synchronization between beams and synchronization in anti-phase between beams and the hub is observed. Whereas, at the second resonance peak full synchronization in phase can be seen between beams and the hub. The same scenario of synchronization phenomenon is observed also in de-tuned rotors at the first resonance peak. Moreover, the full synchronization appeared in case of the second resonance in the symmetric model and the one with shorter beam. Furthermore, the localization of the vibrations is noticed in the de-tuned rotors, model with thicker beam and with shorter beam. Only in the rotating structure with one longer beam at the second resonance, the localization of vibration is observed in the two similar beams. Compering time series from numerical simulations of de-tune models and experimental studies one can observe, that beams are synchronized with a difference of the magnitude. Based on the scenario of synchronization can be 
Fig. 20 Photos from high-speed camera for the deformations corresponding to the maximum swing amplitudes frequency $5.9 \mathrm{~Hz}$,
Fig. 21 Photos from high-speed camera for the frequency $19.7 \mathrm{~Hz}$ deformations corresponding to the maximum swing amplitudes
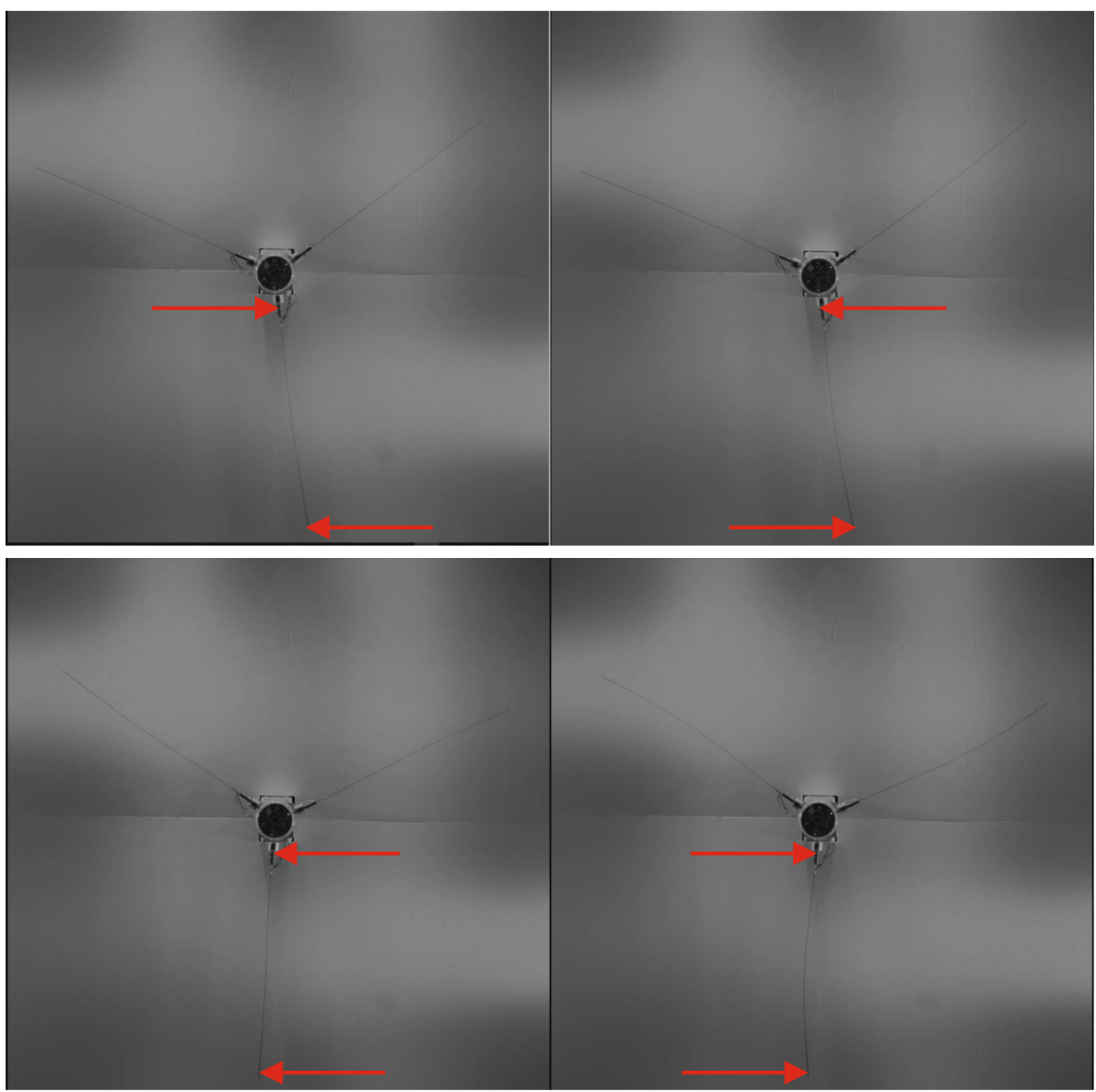

concluded that in the blades in the laboratory stand are slightly de-tune.

Acknowledgements The work is financially supported by grant 2016/23/B/ST8/01865 by Polish National Science Centre, Poland.

Open Access This article is licensed under a Creative Commons Attribution 4.0 International License, which permits use, sharing, adaptation, distribution and reproduction in any medium or format, as long as you give appropriate credit to the original author(s) and the source, provide a link to the Creative Commons licence, and indicate if changes were made. The images or other third party material in this article are included in the article's Creative Commons licence, unless indicated otherwise in a credit line to the material. If material is not included in the article's Creative Commons licence and your intended use is not permitted by statutory regulation or exceeds the permitted use, you will need to obtain permission directly from the copyright holder. To view a copy of this licence, visit http://creativecomm ons.org/licenses/by/4.0/.

\section{References}

1. P. Alevras, D. Yurchenko, A. Naess, Meccanica, 49 (2014)

2. I. Blekhman, Synchronization in Science and Technology (ASME, New York, 1988)
3. N.K. Chandiramani, L. Librescu, C.D. Shete, Aerosp. Sci. Technol. 6, 8 (2002)

4. M.T.M. Crespo da Silva, Int. J. Solids Struct. 35, 7-8 (1998)

5. K. Czolczynski, P. Perlikowski, A. Stefanski, T. Kapitaniak, Commun. Nonlinear Sci. Numer. Simul. 17(9), $427(2012)$

6. D. Dudkowski, K. Czolczynski, T. Kapitaniak, Nonlinear Dyn, 95 (2019)

7. D. Dudkowski, P. Jaros, K. Czolczynski, T. Kapitaniak, Nonlinear Dyn, 102 (2020)

8. C. Huygens, F. Muguet, Paris (1673)

9. M. Kapitaniak, K. Czolczynski, P. Perlikowski, A. Stefanski, T. Kapitaniak, Phys. Rep. 517(1-2), 1-69 (2012)

10. M. Kapitaniak, P. Perlikowski, T. Kapitaniak, Commun. Nonlinear Sci. Numer. Simul. 18(2) (2013)

11. L. Kloda, S. Lenci, J. Warminski, Int. J. Mech. Sci., 178 (2020)

12. J. Latalski, J. Warminski, G. Rega, Math. Mech. Solids 22, 6 (2017)

13. L. Marcheggiani, R. Chacon, S. Lenci, Eur. Phys. J. Spec. Topics 223(4), 729-756 (2014)

14. E. Manoach, J. Warminski, L. Kloda, A. Teter, MATEC Web Conf., 83 (2016)

15. E. Manoach, J. Warminski, L. Kloda, A. Teter, Compos. Struct., 170 (2017)

16. A. Najdecka, T. Kapitaniak, M. Wiercigroch, Int. J. Non Linear Mech., 70 (2015)

17. S.K. Sinha, J. Sound Vib. 332, 9 (2013)

18. Z. Szmit, J. Warminski, MATEC Web Conf., 148 (2018) 
19. Z. Szmit, J. Warminski, AIP Conf. Proc., 1922 (2018)

20. P. Vannucci, G. Verchery, Compos. Struct. 58, 1 (2002)

21. J. Warminski, L. Kloda, J. Latalski, A. Mitura, M.Kowalczuk, Nonlinear Dyn, 103 (2021)

22. J. Warminski, L. Kloda, S. Lenci, Meccanica, 55 (2020)

23. J. Warminski, J. Latalski, Procedia Eng., 144 (2016)

24. J. Warminski, J. Latalski, Int. J. Struct. Stab. Dyn. 17, 5 (2017)

25. J. Warminski, J. Latalski, Z. Szmit, J. Ther. Appl. Mech. 56, 2 (2018)
26. J. Warminski, Z. Szmit, J. Latalski, Eur. Phys. J. Spec. Topics 223, 4 (2014)

27. K. Wiesenfeld, Eur. Phys. J. Spec. Topics 223(4), 687696 (2014)

28. B. Witkowski, P. Perlikowski, A. Prasad, T. Kapitaniak, Eur. Phys. J. Spec. Topics 223, 707-720 (2014) 